\title{
High-energy accelerators above pulsar polar caps
}

\author{
R. X. Xu, G. J. Qiao, and B. Zhang \\ CAS-PKU joint Beijing Astrophysical Center and Department of \\ Astronomy, Peking University, Beijing 100781, China
}

\begin{abstract}
Similar to the terrestrial collision accelerators of $\mathrm{e}^{ \pm}$, another kind of accelerator is above a positively or negatively charged pulsar polar cap. In the case of pulsars with magnetic axis parallel (anti-parallel) to rotational axis, relativistic $\mathrm{e}^{+}\left(\mathrm{e}^{-}\right)$with Lorentz factor $\gamma \sim 10^{6}$ hit the electrons in the polar caps. These scenarios are investigated both for pulsars being BSSs (bare strange stars) and for pulsars being NSs (neutron stars). Such a study may be valuable to differentiate NSs and BSSs observationally.
\end{abstract}

High-energy electron-positron collision in laboratory, where the energy in the center of mass $\left(E_{\mathrm{cm}}\right)$ is about $\mathrm{GeV}$, has been successfully used to study the structure of elementary particles and their interactions. For anti-pulsars, when a backflow $\mathrm{e}^{+}$beats an electron in polar cap, $E_{\mathrm{cm}}$ is also about $\mathrm{GeV} . E_{\mathrm{cm}}=$ $0.511 \mathrm{MeV} \sqrt{2 \gamma} \sim 1 \mathrm{GeV}$ for $\gamma \sim 10^{6}$. Therefore, the physics in the polar cap accelerators does not go beyond that in laboratory.

For rotation-powered pulsars, the rotation energy is converted into the kinetic and radiative energy via an induced electric field around a magnetized rotator, hence, copious $\mathrm{e}^{\ddagger}$ in the open field lines will be accelerated in different directions. The outflow produces a power law radiation, while the backflow heats the polar cap. Therefore, nearly half the loss of rotation energy, $\dot{E}=-9.6 \times 10^{30} P_{1}^{-4} R_{6}^{6} B_{12}^{2}$ ergs $/ \mathrm{s}$, is in outflow and backflow. In fact, relativistic backflow particles radiate away part of their energy before reaching the cap. Considering the polar gap and the out gap accelerations, one can find the residual energy of a charged particles striking the cap ${ }^{1}$ being $\sim 5.9$ ergs (thus $\gamma \sim 10^{6}$ ). With a Goldreich-Julian current bombardment, the polar cap will receive an energy rate ${ }^{1} E_{\text {rate }} \sim 8.1 \times 10^{30} P_{1}^{-5 / 3} B_{12} R_{6}^{3} \mathrm{ergs} / \mathrm{s}$, which is in order of $\dot{E}$. The cap has a radius of $r_{\mathrm{p}}=1.45 \times 10^{4} R_{6}^{3 / 2} P_{1}^{-1 / 2} \mathrm{~cm}$, thus the energy flux received is $F_{\mathrm{p}}=1.8 \times 10^{22} B_{12} P_{1}^{-2 / 3} \mathrm{ergs} \cdot \mathrm{s}^{-1} \cdot \mathrm{cm}^{-2}$.

It was believed that NSs and SSs (strange stars) can be differentiated observationally by studying the global cooling behaviors. However, it is found that SSs cool significantly more rapidly than NSs within the first $\sim 30 \mathrm{yr}$ after birth ${ }^{2}$, which makes NSs and SSs almost un-distinguishable based only on the global thermal radiation. Now that pulsars may be BSSs (see, e.g., Xu, Qiao, \& Zhang in this proceeding), and the materials in the polar caps of NSs and BSSs are very different, we suggest to study the local thermal behavior in the polar caps in order to distinguish NSs and SSs. Such suggestion avoids involving us in the detailed microphysics processes in the interiors of NSs and SSs.

BSSs: have cooler polar caps? As a polar cap is hotter than the other part in a pulsar's surface, heat flows from the cap to the equator. If pulsars are 
NSs, such heat flow is negligible, and an electromagnetic shower produced by incident particles can almost be converted into thermal radiation re-radiated. If there is no thermal conductivity, the temperature in the cap is $T_{0}=\left(\frac{F_{\mathrm{P}}}{\sigma}\right)^{1 / 4} \sim$ $4.22 \times 10^{6} \mathrm{~K}$. The coefficient of thermal conductivity mainly due to the transport of heat by electrons in NS surface is given by ${ }^{3} \kappa^{\mathrm{NS}}=3.8 \times 10^{14} \rho_{5}^{4 / 3} \mathrm{ergs} \cdot \mathrm{s}^{-1}$. $\mathrm{cm}^{-1} \cdot \mathrm{K}^{-1}\left(\rho_{5}\right.$ is the density in unit of $10^{5} \mathrm{~g} \mathrm{~cm}^{-3}$ ), which are nearly independent of the details of the lattice. The temperature gradient in the crust of a NS is $\nabla T^{\mathrm{NS}} \sim \frac{T_{0}}{r_{\mathrm{p}}}$, then the heat flow $H^{\mathrm{NS}} \sim \kappa^{\mathrm{NS}} \nabla T^{\mathrm{NS}} r_{\mathrm{p}}^{n} \sim \kappa^{\mathrm{NS}} T_{0} \sqrt{r_{\mathrm{p}}} \sim 1.9 \times 10^{23}$ ergs/s $\ll E_{\text {rate }}(n=1 \sim 2$ when the thermal conduction geometry near polar cap is considered, we let $n=1.5$ here).

However, if pulsars are BSSs, the electron number density in the surface of a BSS, $n^{\text {BSS }}=1.5 \times 10^{34} \mathrm{~cm}^{-3}$, is much lager than that of an NS, $n^{\mathrm{NS}}=$ $2.8 \times 10^{28} \rho_{5} \mathrm{~cm}^{-3}$. Hence, the coefficient of thermal conductivity in BSS surface, $\kappa^{\mathrm{SS}} \sim\left(\frac{n^{\mathrm{SS}}}{n^{\mathrm{NS}}}\right)^{4 / 3} \kappa^{\mathrm{NS}} \sim 4.35 \times 10^{7} \kappa^{\mathrm{NS}}$, although there is no detailed calculation of $\kappa^{\mathrm{SS}}$ in literature. Therefore the correspondent heat flow in BSS, $H^{\mathrm{SS}} \sim 8.3 \times 10^{30}$ ergs/s, is comparable with $E_{\text {rate }}$, being un-negligible. Such heat flow will result in a much lower polar cap temperature in bare strange stars.

Anti-pulsars with $\boldsymbol{\Omega} \cdot \mathbf{B}>0$ Electrons are pulled out, while positrons bombard the cap. Such process is very similar to that in the laboratory collision of $\mathrm{e}^{ \pm}: \mathrm{e}^{+} \mathrm{e}^{-} \rightarrow \mathrm{f} \overline{\mathrm{f}}\left(\mathrm{e}^{+} \mathrm{e}^{-}\right), \gamma \gamma(\mathrm{f} \overline{\mathrm{f}}$ is a pair of fermion). A gauss-like peak $\sim 0.511$ $\mathrm{MeV}$ spectrum could be observed. However, if pulsars are NSs, additional bremsstrahlung may produces a lower continue spectrum superposed on the peak spectrum. Therefore, NSs and BSSs may be distinguished by spectrum observations.

Pulsars with $\Omega \cdot \mathrm{B}<0 \quad$ Positive charged particles are pulled out, while an electron shower pours on the cap. If pulsars are $\mathrm{NSs}$, the pulled ions being $\mathrm{Fe}$ and/or He nucleus (the composition of the cap surface is uncertain) might result in a formation of line spectrum in $0.04 \mathrm{keV} \sim 10 \mathrm{keV}$ according to the energy levels of atom $E_{n}=-13.6 \mathrm{eV} Z^{2} \frac{1}{n^{2}}$. No such line can be observed if pulsars are BSSs. Observationally, there is no convinced signature of ion line in rotation-powered pulsars. Hence, such pulsars may tend to be BSSs. Perhaps, the future missions of Astro- $E$ (with a fine resolution spectroscope in $0.4-10$ $\mathrm{keV}$ ) would make it clear, that heavy ion (such as iron) lines can or cannot be seen from rotation-powered pulsars.

Conclusion Two conclusions: 1. The polar cap of bare strange star may be cooler than that of neutron star; 2 . Spectrum observation might tell that a pulsar is a bare strange star or a neutron star.

We thank Dr. J.L. Han, Mr. B.H. Hong, and other members in our pulsar group. This work is supported by NSFC (No. 19803001, N0.19910211260-570A03), by the Climbing project of China, by Doctoral Program Foundation of Institution of Higher Education in China and by the Youth Foundation of PKU.

References Wang, F., et al. 1998, ApJ, 498, 373

Schaab, C., et al. 1997, ApJ, 480, L111

Jones, P. B. 1978, MNRAS, 184, 807 